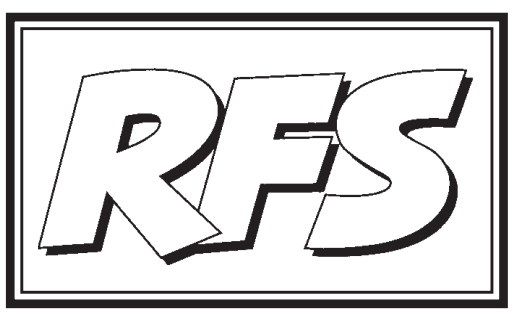

Revista de Fomento Social, 59 (2004), 395-408

\title{
La formación de recursos humanos para la cooperación internacional ${ }^{1}$
}

Carmelo ANGULO BARTUREN²

(PALABRAS CLAVE: FoRMACIÓN PARA LA COOPERACIÓN, COOPERANTE DEL NORTE, ESTUDIOS DE COOPERACIÓN.

KEY WORDS: TRAINING FOR CO-OPERATION, AGENTS FROM THE NORTH,

STUDIES FOR CO-OPERATION)

1. Introducción

Quiero iniciar esta presentación agradeciendo la invitación de ACADE, que llega en un momento muy prometedor de la cooperación en España, alentado por la llegada del gobierno socialista, y al fin de uno de los ciclos más sustantivos y esclarecedores de mi vida profesional tras casi ocho años de trabajo en las Naciones Unidas.

Texto de la ponencia de las II Jornadas de ACADE (14 de mayo de 2004). Se ha conservado el estilo oral propio de dicha intervención.

2 En la fecha de la ponencia, Asesor del Programa de Naciones Unidas para el Desarrollo (PNUD). 
Permítanme decirles, antes de empezar, que el momento que vive la cooperación internacional es, por una parte, de languidecimiento, por otra, de perplejidad y, finalmente, sólo por parte de un núcleo destacado de países, de antimultilateralismo. Languidece el sistema porque los recursos son escasos en proporción a la riqueza en manos de los países, las empresas y los particulares. Aquellos, medidos en proporción al PIB, cayeron a la mitad de su nivel de mediados de los 70 , de 0,46 a 0,23 . Tampoco se ha producido un verdadero engarce del sector privado con ejes vitales de desarrollo y debajo de inversiones, de créditos blandos, microcréditos y transferencias de tecnologías, apenas asoman algunos gestos de responsabilidad empresarial en el área social. Por su parte, la sociedad civil organizada ha crecido desmesuradamente representando una enorme variedad de sensibilidades y una fragmentación de intereses y metodologías a la hora de acercarse a la realidad del desarrollo.

Por otra parte, y esto hace a la perplejidad, a pesar del avance espectacular de la democracia, en todo el planeta y en los Países en Vías de Desarrollo (PVD), al menos en sus aspectos formales y electorales, hay un cuestionamiento casi generalizado sobre la manera de operar de los partidos y las instituciones, una especie de vaciamiento de la política con el corolario del descrédito ciudadano. Los Estados, reformulados al amparo de las rígidas reglas del consenso de Washington, más que nunca, parecen incapaces de democratizar y generar cohesión social, al estar desprovistos de poder o utilizarlo errónea o corruptamente, o al ser sometidos a controles de superávit fiscal y a reglas de juego que apenas permiten la inversión social, la modernización tecnológica y la generación de reglas de juego previsibles. A los presidentes (Argentina, Bolivia, Haití) se les saca del poder abruptamente con manifestaciones o revueltas populares no demasiado sofisticadas.

Es el universo que veo desde hace una larga década en la América Latina de la crisis cíclica, la de la inestabilidad contenida a duras penas y del malestar y descontento ciudadano con el rompimiento de promesas electorales, la falta de acceso masivo a servicios básicos y los daños directos y colaterales de la globalización. Una globalización que restringe el comercio, impone patrones culturales ajenos y, para colmo, aleja el control interno de variables como precios, reglas fitosanitarias, cuotas de acceso a mercados... Sin hablar de la deuda, que es ya más eterna que externa.

Y todo esto, que nos hervía la sangre hace unos años, ahora que nos encontramos en la cresta de la ola, lo apreciamos, aquí y muchas veces allí, con cierta frialdad, distancia y como decía recientemente Moisés Naim, en una reciente conferencia, "sin memoria, sin esperanza, instalados en el presente". El cambio no es pequeño porque ahora somos, además, parte delas decisiones y culpables, por ejemplo, de los incumplimientos de los protocolos de Kyoto, de las promesas de la Cumbre de Monterrey y de seguir llamando crédito al desarrollo a lo que muchas veces son puramente ayudas encubiertas a la exportación o a la internacionalización de nuestras empresas.

Este pequeño botón de muestra, que explico sólo de manera superficial, ha sido agrandado por la catástrofe humanitaria de Irak, refiriéndome al sesgo antimultilateral, que ha erosionado sin límites el sistema internacional, desviado recursos previamente asignados a la cooperación o el desarrollo, devaluado a la ONU y puesto de rodillas a los principios del derecho humanitario. Ése es el mundo y el contexto con el que nuestros jóvenes y mayores que acceden a la cooperación van a encontrase aquí, en los territorios del Norte, y allí, en las dehesas y páramos de ese Sur, cada vez más ancho e interminable. Por ello, propongo, sin solemnidades, que es el momento de pensar en una formación para el desarrollo más agresiva y militante, más transgresora del modelo mayoritario y conformista, de visión asistencialista y rentista, que aún promociona valores, principios, instrumentos y metodologías fuertemente cuestionadas o alteradas por el uso. Algunas ideas que el sistema de formación debe transmitir, son entre otras:

A) Ante tanta pobreza, a la que se suma la creciente desigualdad, explosiva en sus derivaciones, ante tanto conflicto incomprensible e indefendible legalmente, hay obligatoriamente que volver con más insistencia que nunca a la cultura de paz, a las metodologías y proyectos de solución de conflictos, a la instalación masiva del diálogo y la concertación como armas de superación de las tensiones y al arbitraje, la mediación y los buenos oficios. Y no sólo entre países o regiones, entre religiones y credos, sino al interior de los Estados en los que trabajamos. ¿Es posible una cooperación eficiente en el conflicto, aunque esta lo pueda paliar? ¿Hay algo que se pueda oponer a la intervención bélica preventiva que no sea un agresivo pacifismo preventivo?

B) La pobreza actual es injusta e insoportable y, además, para la versión egoísta del mundo y el mercado comienza a ser el peor negocio posible. Son necesarios no sólo objetivos y metas globales y compartidas, para los que los Objetivos del milenio (ODM) sirven más que adecuadamente, sino acciones de urgencia bien sea a través de Fondos (hambre), tasas 
a las transacciones o impuestos especiales para cerrar urgentemente la brecha, condonaciones exhaustivas de la deuda (la iniciativa HIPIC no alcanza) y hacer llegar masivamente medicamentos antivirales a los sectores y países más necesitados, no productores. También podría servir cambiar abruptamente las reglas de juego del comercio, terminando los subsidios.

C) La democracia plena, la democracia de ciudadanía, debe de ser el otro gran objetivo de la cooperación. Los gobiernos y muchos actores internos y externos han acabado acomodándose a democracias de baja calidad e intensidad. Sin instituciones y partidos creíbles, sin Estado de derecho, sin transparencia y sin fiscalización, o monitoreo ciudadano, no hay democracia plena y la cooperación internacional es, obviamente, un juguete al viento.

D) Me pregunto qué se puede hacer frente a esta brutal brecha tecnológica que concentra casi exhaustivamente Internet en los países de la OCDE (80\%), que hace prácticamente imposible la compra de un ordenador a un salario intermedio en África, que tiene precios inalcanzables para los medicamentos de lucha contra el VIH-SIDA y que restringe el acceso telefónico, eléctrico y del agua a sectores privilegiados de los PVD.

E) Sin cumplir urgentemente las promesas de la cumbre de Monterrey y sin aumentar la cantidad y la calidad de la ayuda, hay realmente pocas esperanzas. Frente a los flujos de inversión en tecnologías, los capitales golondrinas o las transacciones ilegales de la droga (700.000 mil millones de dólares de EEUU por año), los flujos de cooperación neta son casi ridículos e insignificantes.

F) Por fin, sin una ONU, ésta u otra diferente que se debiera crear, es decir, sin un mínimo sistema internacional, con los organismos de apoyo necesarios, es difícil pensar en regulaciones y respuestas masivas a las emergencias, los posconflictos, las grandes enfermedades, los crímenes de lesa humanidad y el control del tráfico ilegal de estupefacientes, personas y flujos monetarios.

Sin ánimo de ser exhaustivo, tengo que reconocer que lo que se nos pide a los trabajadores y profesionales de la cooperación es que seamos, al mismo tiempo, expertos en pobreza, deuda, gobernabilidad social y política, en deuda

3 Highly Indebted Poor Countries Initiative. y comercio y en nuevas tecnologías. Además debemos ser movilizadores y planificadores de recursos, que son evaluados y fiscalizados exhaustivamente, expertos en emergencias y catástrofes y, finalmente, componedores de diálogos y consensos, no sólo con nuestras contrapartes habituales sino con nuestros amigos de agencias de cooperación y ONGD con los que preferimos muchas veces competir antes que coordinar, aunque nuestras agendas y diagnósticos se parezcan como gotas de agua.

Ante tanta complejidad espero que no cunda el pánico. Somos legión los que seguimos luchando por un mundo mejor y más justo, muchas redes, muchas alianzas, muchas almas juntas. No hemos podido cambiar este mundo pero este mundo tampoco nos convence y rehusamos a que nos cambie a nosotros. Déjenme en este contexto, que tiene que ser emocional y racional al mismo tiempo, dar algunas ideas de cómo enfrentar la formación para la cooperación en nuestros días.

\section{Los mensajes iniciales desde el Norte}

El reto es que nuestros técnicos, expertos y voluntarios conozcan a fondo el sistema global internacional actual con su presente arquitectura y sus indicadores y tesis principales, fundamentalmente para presionar y empujar hacia su transformación. Se trata de cambiar el norte mientras se facilita el cambio en el sur. Los vetos del Consejo de Seguridad, los votos ponderados de las Instituciones financieras internacionales (IFIS), las restricciones al comercio y los criterios actuales de reducción de deuda, son injustos, perversos o precarios (o las tres cosas a la vez). Si bien reconstruir una nueva arquitectura financiera y legal internacional tiene que tomar tiempo, hay poca discusión y presión pública para un cambio que reclama a voces el fracaso del llamado consenso de Washington. Desde las aulas deben aprenderse los valores, argumentos y elementos del nuevo sistema que hay que promover no sólo globalmente (G8, UE, IFIS etc) sino además en cada reunión local y nacional para construir nuevos proyectos HIPIC, compromisos concretos para alcanzar los ODM y marcos de cooperación innovadores con autoridades nacionales y locales.

Los que tiene que desplazarse y trabajar en el Sur, además tienen que prepararse con mentalidad de orfebre no de apostador exitoso. Los cambios de valores y los institucionales, suelen ser largos procesos, a veces genera- 
ciones, por lo que es tan importante poner semillas y cavar el surco como recoger el fruto. Creo más en los constructores de catedrales, pacientes y sólidos, que en los malabaristas que tornan chisteras por conejos instantáneamente. La generación de valores (cultura del trabajo, de paz, diálogo y participación), el fortalecimiento de la institucionalidad (justicia, aduana, comercio, compras y rentas) y la descentralización son pilares fundamentales de asiento democrático y del desarrollo humano pero necesitan un horizonte político. Estos, junto a la frontal lucha contra la pobreza, la defensa del medio ambiente y la lucha contra la discriminación, definen y precisan de una preparación muy especializada y de una mística y un compromiso especial que se debe aprender y exigir como piezas básicas del desarrollo sostenible. Pero el desarrollo es como un árbol, debe crecer de abajo arriba y éste es un trabajo de orfebres, sutiles artistas y pacientes negociadores.

En otra instancia, los que reciben cooperación y que no sólo trabajan como contrapartes con nosotros sino que se forman a menudo entre nosotros conocen esta industria del desarrollo y la Cooperación internacional y sus diferentes andariveles. Pero ellos y nosotros, tenemos juntos que abordar la necesidad de conocer y hacer transparente el Estado y las ayudas que pueda recibir, fomentar el reto del empoderamiento (autoestima, identidad y liderazgo), la ventaja del diálogo, la participación de la mujer y la necesidad de alianzas con los demás actores para achicar los tiempos y minimizar los impactos negativos. La cultura de prevención en general y la concepción de la reducción de las vulnerabilidades socionaturales son decisivas en sus procesos formativos.

Estos tres tramos de principios que acabo de mencionar: 1) conocimiento y presión hacia una nueva arquitectura y reglas del juego, 2) la generación de valores tendentes a crear institucionalidad democrática, luchar contra la discriminación y las vulnerabilidades y 3) las metodologías de diálogo, empoderamiento y participación. Estos principios constituyen, a mi modo de ver, el núcleo central formador de los componentes sustantivos pero refuerzan también el terreno de las actitudes y los principios con los que el trabajador de la cooperación se mueve. Son los que generan los resortes de la serena trasgresión frente al injusto orden internacional, al mismo tiempo que buscan la manera de remover el autoritarismo y el clientelismo, los que mueven al incorformismo frente a la marginación, la indigencia y la polarización social con las que frecuentemente nos encontramos, los que orientan hacia políticas públicas más justas y los que nos hacen solidarios con los grupos más vulnerables. El experto en cooperación, si quiere ser eficiente, es un tomador de riesgo por naturaleza, es un adivinador e intérprete de contextos, debe ser un fino analista y es un inspirador y promotor de valores, políticas publicas y metodologías.

\section{Los dilemas de la formación de la cooperación internacional}

En la actualidad, la formación dirigida a la Cooperación internacional se enfrenta a una serie de dilemas entre los que destaco los siguientes.

El primero pretende fortalecer las actitudes (modestia, coraje y compromiso) frente a las aptitudes (conocimientos y habilidades) o a la inversa Las primeras son absolutamente necesarias, para mí son una condición sine qua non. Las segundas son relevantes pero la cooperación (quizás no el comercio) necesita corazón y pasión, ya que lo que se transmite y lo que cambia es muchas veces intangible en el corto y medio plazo.

El segundo es preparar para cambiar y reformar o para inspirar, incentivar y acompañar. Hay mucho modelo preconcebido de cómo hacer las cosas (clonación o copia adaptada) y la realidad es tozuda y todo cambio debe inspirarse localmente.

Otro dilema habitual es orientarse hacia élites o instituciones centrales o líderes de base, locales o comunitarios. La tentación constante por su visibilidad y proximidad es trabajar con líderes de élite cercanas a nuestras contrapartes habituales (capitalinas y deformación académica) y desconocer o minusvalorar a los líderes de base autóctona, campesina o de pequeñas y medianas empresas que pueden, desde los escenarios locales y provinciales, cambiar las cosas y dotar de nueva energía a las propuestas y las políticas. La preparación para descubrir y formar líderes es una de las más descuidadas por la Cooperación internacional y las fuerzas políticas.

Trabajar con gobierno e instituciones o a través de ONGD, sector privado o entidades descentralizadas. Hay como fijaciones y reparto de cotos de caza. Puede servir cualquiera de ellos pero fomentando la coordinación y refiriéndonos siempre a planes estratégicos o prioridades de desarrollo nacional.

Trabajar hacia y para la reducción de la pobreza, directamente con los pobres, olvidando que sin instituciones no hay gerencia pública ni calidad en las respuestas y que la solución de la inequidad y la reducción de las vulnerabilidades, pasa generalmente, por su volumen y complejidad, por la acción del Estado. 
Actuar solos o asociados. Es fundamental pensar en plataformas, campañas globales compartidas, esfuerzos de cofinanciación, asociación con Organismos internacionales, es decir, coordinación versus aislamiento y fragmentación.

Todos estos dilemas constituyen valores y antivalores para mí, y cada uno de ellos requiere de formación y prácticas específicas. Tienen su lenguaje corporal y su jerga y metodologías que merecen ser tratadas con simulaciones y con ejercicios y prácticas vigiladas allí sobre el terreno.

\section{La cooperación requiere habilidades gerenciales}

Además de actitudes, conocimientos y preocupaciones sustantivas, hoy más que nunca, precisamos de habilidades gerenciales. La planificación y gestión del desarrollo y la cooperación internacional, precisan hoy de, al menos, cinco capacidades netas:

- Planificación estratégica (prioridades compartidas y calendarizadas), establecimiento de objetivos, medios y riesgos claros (metodología del marco lógico) y definición de fortalezas y debilidades (matriz $\mathrm{DAFO}^{4}$ ). Estos y otros elementos tienen que ver con la definición del problema y al contexto dinámico en el que vamos a movernos.

- Sistemas de alarma, medición e indicadores, que hacen referencia a la eficiencia y efectividad de nuestro trabajo respecto a objetivos y gastos.

- Selección, contratación y formación de recursos humanos que van a ser decisivas para la continuidad del esfuerzo y la diversidad de funciones. Esto lleva a innovaciones organizativas permanentes y hace fundamental los programas de formación permanente.

- Mecánicas de compras y licitaciones, que suelen ser elementos cuestionados de la transparencia de los proyectos.

— Estrategias de financiación y movilización de recursos, que generalmente son la base del trabajo en equipo, plataforma o en red (networking), tan habitual en las transacciones de cooperación.

Si quisiéramos ponerlo de otra manera, podríamos organizar la formación en torno a cinco pilares:

4 Debilidades, amenazas, fortalezas y oportunidades. a) La creación de valores de paz, diálogoy concertación como mecanismos de una cultura más amplia de prevención y superación de los desgarros de las guerras y sus secuelas socioambientales. Las técnicas de negociación, mediación, consenso, cabildeo (advocacy) y comunicación para el desarrollo, habilitadas con los respectivos enfoques de identidad, cultura, religión e idioma, son esenciales.

b) La profundización de la formación en la transversalidad de género, medio ambiente, participación, prevención y riesgos socioambientales y seguridad humana (acceso a alimentos, seguridad física y empleo decente).

c) La movilización de recursos y voluntades, que está estrechamente unida a la financiación del desarrollo, la generación de alianzas y plataformas, la coordinación de actores y, de nuevo, a la información y la comunicación pública. Puntos fuertes son las complejas y, a veces, decisivas mecánicas de cumbres de NNUU, mesas de donantes, grupos consultivos, etc

d) La gestión del desarrollo que se vincula, en la fase de diseño y ejecución, a procedimientos, contratos, compras, formación de recursos humanos, presupuesto y gasto, normas y ventajas impositivas, uso de nuevas tecnologías y fijación de mecanismos de medición y monitoreo. Y en la fase ex post, a evaluaciones y auditorías, hoy totalmente preceptivas en todas partes.

e) La promoción de los marcos legales y constitucionales, los estatutos profesionales y el marco genérico de deberes y derechos de los técnicos y expertos (entitlements)

\section{Análisis crítico de los actuales estudios de cooperación}

Aunque me moveré con enorme prudencia, ya que estoy alejado, veo un poco lo que pasa en España y afuera. Algunas observaciones sobre patrones que se repiten.

- Hay proliferación de seminarios, encuentros, diplomas y maestrías de cooperación internacional tanto en centros públicos como privados. Uno se pregunta si hay tanta demanda aquí y si es necesario enviar a tanta gente allí. ¿Se trata de formar masas críticas o de generar profesionales solventes? ¿Se distingue bien entre formar para la filantropía y el 
asistencialismo o formar para la cooperación que impacta al desarrollo y transforma conductas y estructuras?

- No siempre se utiliza una secuencia lógica, pasando de lo general a lo particular, de lo teórico a lo práctico, de lo valorativo a lo metodológico. Uno se pregunta si hay verdaderos enseñantes especializados en cooperación (asignatura que no existe en las facultades ni cátedras prácticamente), o si forman sólo los que vienen de ciencias conexas. Si se puede formar sólo con profesores del Norte y sin apenas profesionales y enseñantes formados en el Sur.

- Los cursos ofrecidos se presentan como una sucesión de seminarios, conferencias y eventos, más o menos concatenados, en donde prima la disponibilidad de los invitados y sus propios requerimientos. Más que cursos o maestrías especializadas en temas o áreas, en las que se brinda con excelencia el mejor conocimiento en alguna materia, se trata de agrupaciones de familias de pensamiento, círculos de reciprocidad y feudos de escuelas de Relaciones Internacionales, Derecho Internacional, Economía o Sociología Política. La duda es si se trata de redes verdaderas.

- Se pone por lo general un énfasis excesivo en la cooperación no gubernamental, descuidando a menudo la importancia del sector público, de las instituciones internacionales, y del sector empresarial para la formulación e implementación de políticas transformadoras, capaces de dinamizar las energías sociales y promover el cambio económico y político a gran escala $\left(\mathrm{ODM} \mathrm{n}^{\circ} 8\right)$. Los cursos parecen responder más a las necesidades en materia de gestión de recursos humanos de las ONGDs que a las grandes prioridades globales en materia de desarrollo humano.

- Si bien se dan niveles de exigencia razonables dentro de una gran flexibilidad de metodologías para alcanzar títulos (prácticas, tesinas créditos etc), no parece haber criterios de medición de excelencia y calibración de conocimientos de los asistentes y profesionales egresados, ni mecanismos que faciliten realizar comparaciones objetivas de las diferentes ofertas. En este terreno se impone ir elevando los niveles de exigencia que acrediten que los graduados cuentan con la actitud y aptitud necesarias para afrontar el desempeño profesional en un contexto muy exigente y muy arriesgado desde el punto de vista de la seguridad física.
— La búsqueda de financiación específica es clave para la apertura y sostenibilidad de los cursos y maestrías. Son muy pocas las Universidades que las brindan como una opción vocacional financiada con carácter ordinario, dependiendo en gran medida del mercado. En su defecto, las expectativas de consolidar las diversas ofertas de formación depende tanto de la capacidad de los diversos equipos organizadores para obtener financiación externa, asociándose a instituciones muy diversas, como de las expectativas de ingresos por matrícula (Riesgo de calidad).

— Las oportunidades de inserción profesional de los postgraduados tienen por lo general un carácter marcadamente nacional en cuanto al reclutamiento lo que dificulta su aceptación internacional. Los estudiantes de nacionalidad extranjera se ven a menudo discriminados en el acceso a os programas de prácticas y a las oportunidades de desarrollo laboral. Por ello, la internacionalización de nuestros campus, y la creciente presencia de estudiantes extranjeros, debe ir acompañada de un mayor esfuerzo en materia de incentivos para los estudiantes extranjeros y de internacionalización de las oportunidades.

- Se impone en consecuencia una definición curricular más formalizada, el fortalecimiento de los centros especializados en sectores y metodologías, el incremento de la investigación teórica y aplicada, el impulso de las publicaciones especializadas de difusión internacional, la agrupación de los cursos formales en asociaciones y plataformas de validación, y las alianzas con centros extranjeros especializados de los PVD que permitan intercambios de profesores, expertos y alumnos.

- Las iniciativas más creativas y originales encuentran, a menudo, importantes obstáculos para su materialización debido a la centralización característica de la gestión universitaria y de sus procedimientos de control administrativo. El proceso de convergencia europea en materia de educación superior arroja, por lo demás, importantes incertidumbres sobre las posibilidades de mantener la situación actual, e impone la necesidad de adoptar a corto y medio plazo cambios radicales en el modelo actual de formación de postgrado.

- Así las cosas, no se debe descartar la procedencia de crear una Escuela Nacional de Cooperación, cuya titulación o validación de estudios pudiera dar pie, sin perjuicio del mantenimiento y renovación del modelo actual de formación a través de diversas instituciones públicas y privadas, a una formalización del estatus del cooperante y experto en la 
función pública, tanto a nivel central como autonómico, que asegurara su reconocimiento y competencia profesional.

\section{La experiencia del Master en Cooperación Internacional Descentrali-} zada: Paz y Desarrollo, de la Universidad del País Vasco

Por último, quisiera referirme brevemente a la experiencia adquirida en este terreno por el master en Cooperación Internacional Descentralizada: Paz y Desarrollo, de la Universidad del País Vasco, con el que vengo colaborando desde hace casi una década, y de cuyo Comité Académico tengo la satisfacción de formar parte desde el presente año académico.

Nos situamos frente a las posiciones que no conciben otra forma de cooperación que aquellas que suponen el desmantelamiento de lo público, al situar el comienzo y el final de la cooperación internacional en la cooperación no gubernamental, o que pretenden ignorar la pesada importancia del mercado. Ello está en consonancia con las conclusiones del reciente Informe del PNUD sobre la Democracia en América Latina, en cuya elaboración he tenido el honor de participar. Por eso, nuestro master parte de la convicción de que es necesario revalorizar la importancia de las dimensiones propiamente políticas del desarrollo humano, profundizando el fortalecimiento de la democracia y la ciudadanía social, y la profunda conexión entre paz y desarrollo, como única base sólida para una nueva legitimidad del Estado y de sus instituciones, promoviendo a su vez una mayor diversidad y flexibilidad en la política económica, frente a los esquemas rígidos y deshumanizados que caracterizaron las políticas de corte neoliberal.

A su vez, nuestro master realiza un gran esfuerzo por asegurar un programa de prácticas internacionales de alto nivel con el objetivo de facilitar la inserción laboral de nuestros titulados, mediante la puesta en acción de diversas iniciativas de cooperación con instituciones públicas y privadas muy diversas, y muy especialmente, con el sistema de Naciones Unidas. Al respecto quiero destacar el importante respaldo que ha venido ofreciendo la Dirección de Cooperación al Desarrollo del Gobierno Vasco a nuestro programa de prácticas internacionales en el sistema de las Naciones Unidas. El Gobierno Vasco nos brinda ciertamente su importante apoyo financiero pero toda la organización, gestión e implementación del Programa de Prácticas Internacionales, incluyendo la interlocución y negociación con el Sistema de las Naciones Unidas, corresponde exclusivamente a los responsables académicos de nuestro master, es decir a la comisión académica, y por supuesto a nuestros interlocutores dentro de organismos tales como el PNUD, UNIFEM, UNICEF, o FNUAP ${ }^{5}$, entre otros. La puesta en acción no fue sencilla pero la generosa colaboración de diversas personas del sistema de las $\mathrm{Na}$ ciones Unidas, facilitaron la extensión y el óptimo funcionamiento de este programa de prácticas internacionales, y la consolidación de un mecanismo de cooperación y solidaridad que se ha revelado muy eficaz y con resultados satisfactorios para todas las partes. Todo ello con el objetivo de:

a) Contribuir a la importante labor que desarrollan diferentes programas, agencias y organismos vinculados al sistema de Naciones Unidas, en la promoción del desarrollo humano y sostenible, la protección de los derechos humanos, la lucha contra la impunidad, y la promoción de la gobernabilidad democrática. Todo ello, en el respeto de los principios y disposiciones que recoge la Carta de las Naciones Unidas, así como otros instrumentos jurídicos internacionales.

b) Facilitar la capacitación einserción profesional de jóvenes profesionales, de cualquier nacionalidad, en el sistema internacional de cooperación, mediante su incorporación en un programa de prácticas profesionales de alto nivel y larga duración en el seno del sistema de las Naciones Unidas, que les permita mostrar y desarrollar las actitudes y aptitudes que requiere un mundo profesional tan exigente, y a la vez tan ilusionante, como el de la cooperación internacional.

c) Responder a la demanda existente desde el propio sistema de Naciones Unidas para el establecimiento de nuevas iniciativas de cooperación institucional descentralizada que puedan contribuir al fortalecimiento del sistema internacional de cooperación, y a la maximización de sus recursos y capacidad de acción, mediante el desarrollo de iniciativas originales y creativas, en un momento en el que su propia supervivencia parece estar en cuestión.

Sé que estamos en una tarea gigantesca y nos aproximamos a una nueva ruta. Pero la cooperación debe de recuperar empuje y militancia, no conformarse con su creciente institucionalización y reconocimiento público que nos lleva a vernos hoy como un colectivo especializado. Estoy convencido

5 PNUD = Programa de Naciones Unidas para el Desarrollo. UNIFEM = Fondo de desarrollo de Naciones Unidas para la Mujer. UNICEF = Fondo de Naciones Unidas para la Infancia y la Educacion. FNUAP = Fondo de Naciones Unidas para la Población. 